\title{
Proper Definitions and Demonstration of Dirichlet Conditions
}

\author{
Pushpendra Singh $^{\dagger}$, Amit Singhal ${ }^{\ddagger}$, Binish Fatimah ${ }^{\S}$, \\ Anubha Gupta $\$$, and Shiv Dutt Joshill
}

Fourier theory is the backbone of the area of Signal Processing (SP) and Communication Engineering. However, Fourier series (FS) or Fourier transform (FT) do not exist for some signals that fail to fulfill a predefined set of Dirichlet conditions (DCs). We note a subtle gap in the explanation of these conditions as available in the popular signal processing literature. They lack a certain degree of explanation essential for the proper understanding of the same. For example, the original second Dirichlet condition is the requirement of bounded variations over one time period for the convergence of Fourier Series, where there can be at most infinite but countable number of maxima and minima, and at most infinite but countable number of discontinuities of finite magnitude. However, a large body of the literature replaces this statement with the requirements of finite number of maxima and minima over one time period, and finite number of discontinuities. The latter incorrectly disqualifies some signals from having valid FS representation. Similar problem holds in the description of DCs for the Fourier transform. Likewise, while it is easy to relate the first DC with the finite value of FS or FT coefficients, it is hard to relate the second and third DCs as specified in the signal processing literature with the Fourier representation as to how the failure to satisfy these conditions disqualifies those signals from having valid FS or FT representation.

These concepts are taught worldwide in the standard course of "Signals and Systems" to the second year undergraduate students of Electrical/Electronics/ Communication/Computer Engineering. Lack of clarity in the explanation of DCs in the signal processing textbooks makes it hard for the young engineering students to understand these concepts. In fact, many instructors roll out

*This work has been submitted to the IEEE for possible publication. Copyright may be transferred without notice, after which this version may no longer be accessible.

${ }^{\dagger} \mathrm{P}$. Singh is with the Department of ECE, National Institute of Technology Hamirpur, Hamirpur (HP) India (pushpendrasingh@iitkalumni.org).

${ }^{\ddagger}$ A. Singhal is with the Department of ECE, Bennett University, Greater Noida (UP) India (singhalamit.iitd@gmail.com).

$\S \mathrm{B}$. Fatimah is with the Department of ECE, CMR Institute of Technology, Bengaluru, India (binish.fatimah@gmail.com).

ब A. Gupta is with the Department of ECE, Indraprastha Institute of Information Technology Delhi, Delhi, India (anubha@iiitd.ac.in). ${ }^{\$}$ Corresponding author.

${ }^{\|}$S.D. Joshi is with the Department of EE, Indian Institute of Technology Delhi, Delhi, India (sdjoshi@ee.iitd.ac.in). 
this topic as a "reading assignment" for the students because it may be equally uncomfortable for signal processing instructors to provide suitable explanations for the second and third DCs. In this lecture note, we attempt to provide the required clarifications and provide a lucid but precise description and explanation on DCs. We support the text with the help of suitable examples. The entire signal processing community may benefit from this work. In particular, the presented work can help in teaching these concepts with better clarity to the young signal processing audience.

\section{Relevance}

Fourier theory provides a powerful tool for the analysis of signals. It has been widely used in the areas of signal processing and communication engineering. Fourier representation, such as FS and FT, provides an alternate representation of a time-domain signal in terms of its frequency content that is helpful in the analysis and processing of signals in umpteen number of applications and devices. While FS is evaluated for periodic signals, FT is generally used to analyze non-periodic (or aperiodic) signals, although it can be computed for the periodic signals by roping in impulse functions that are generalized functions.

It is not necessary that every signal will have a valid Fourier representation. In general, it is stated that a signal would have a valid FS or FT representation provided it satisfies a set of conditions given by Dirichlet. These are stated to be sufficient, but not necessary conditions. The Fourier theory and the associated DCs are essential tools that are utilized by the scientific community working in the area of signal processing and related applications. Almost every textbook on "Signals and Systems" as well as many widely-used manuscripts and books [1-10] specify these conditions in the context of Fourier representation and also provide examples, some of which are incorrectly perceived as not fulfilling these conditions.

\section{Preliminaries}

In this section, we present some mathematical preliminaries that are important to understand the concepts with clarity.

Definition 1: A signal $\tilde{x}(t)$ is periodic if, $\tilde{x}\left(t+T_{0}\right)=\tilde{x}(t), \forall t$ where $T_{0}$ is the fundamental period in seconds and $\omega_{0}=\frac{2 \pi}{T_{0}}=2 \pi f_{0}$ is the fundamental frequency in radians/second.

Definition 2: Let $x:[a, b] \rightarrow \mathbb{C}$ be a function and any finite partition of $[a, b]$ is set $P=\left\{a=t_{0}<t_{1}<\cdots<t_{n}=b\right\}$. The variation of $x$ over $[a, b]$ is defined as

$$
\begin{aligned}
V(x,[a, b]) & =\sup \left\{S_{P}: P \text { is a partition of }[a, b]\right\}, \\
\text { where } S_{P} & =\sum_{i=1}^{n}\left|x\left(t_{i}\right)-x\left(t_{i-1}\right)\right| .
\end{aligned}
$$


The function $x$ has 'bounded variation' (BV) on $[a, b]$ denoted as $x \in B V([a, b])$, if $V(x,[a, b])$ is finite, i.e., $V(x,[a, b])<\infty$.

To obtain the variation in (1) the supremum is taken over all possible partitions on $[a, b]$. Some important results related to a function of bounded variation are as follows:

1. If a function $x$ is increasing on $[a, b]$, then $x \in B V([a, b])$ and $V(x,[a, b])=$ $x(b)-x(a)$.

2. If a function $x$ is decreasing on $[a, b]$, then $x \in B V([a, b])$ and $V(x,[a, b])=$ $x(a)-x(b)$.

3. The function $x$ has at most countably infinite discontinuities on $[a, b]$.

4. If $x$ is differentiable and its derivative $x^{\prime}$ is continuous on $[a, b]$, then

$$
V(x,[a, b])=\int_{a}^{b}\left|x^{\prime}(t)\right| \mathrm{d} t .
$$

Next, we illustrate this concept of bounded variation (BV) with a few examples.

\section{Example-1:}

Consider a signal

$$
x_{1}(t)= \begin{cases}\sin (2 \pi / t), & \text { if } t \in(0,1] \\ 0, & \text { otherwise. }\end{cases}
$$

The derivative of $x_{1}(t)$ is given by $x_{1}^{\prime}(t)=-2 \pi \cos (2 \pi / t) / t^{2}$. It is easy to see that $\int_{0}^{1}\left|x_{1}^{\prime}(t)\right| \mathrm{d} t=\infty$. This implies that $V\left(x_{1},[0,1]\right)=\infty$. Hence, signal $x_{1}(t)$ does not have $\mathrm{BV}$ in the time interval $[0,1]$, i.e., $x_{1} \notin B V([0,1])$.

Example-2:

Consider a signal

$$
x_{2}(t)= \begin{cases}t \sin (2 \pi / t), & \text { if } t \in(0,1] \\ 0, & \text { otherwise. }\end{cases}
$$

The derivative of $x_{2}(t)$ is given by $x_{2}^{\prime}(t)=-2 \pi \cos (2 \pi / t) / t+\sin (2 \pi / t) / t$. It is easy to see that $\int_{0}^{1}\left|x_{2}^{\prime}(t)\right| \mathrm{d} t=\infty$. This implies that $V\left(x_{2},[0,1]\right)=\infty$. Hence, signal $x_{2}(t)$ does not have $\mathrm{BV}$ in the time interval $[0,1]$, i.e., $x_{2} \notin B V([0,1])$.

\section{Example-3:}

Consider a signal

$$
x_{3}(t)= \begin{cases}t^{2} \sin (2 \pi / t), & \text { if } t \in(0,1] \\ 0, & \text { otherwise. }\end{cases}
$$


The signal $x_{3}(t)$ has $\mathrm{BV}$ in $[0,1]$ because

$$
\begin{aligned}
V\left(x_{3},(0,1]\right) & =\int_{0}^{1}\left|x_{3}^{\prime}(t)\right| \mathrm{d} t \\
& =\int_{0}^{1}|2 t \sin (2 \pi / t)-2 \pi \cos (2 \pi / t)| \mathrm{d} t \\
& \leq \int_{0}^{1}|2 t \sin (2 \pi / t)|+|2 \pi \cos (1 / t)| \mathrm{d} t \\
& \leq \int_{0}^{1}(2 t+2 \pi) \mathrm{d} t=(1+2 \pi)
\end{aligned}
$$

In fact, one can easily show that the signal $x_{r}(t)=t^{r} \sin (2 \pi / t)$ for $t \in(0,1]$ and zero otherwise, has BV in $[0,1]$ for $r \geq 2$.

\section{Fourier Series and Dirichlet Conditions}

The FS representation of a periodic signal $\tilde{x}(t)$ is defined as

$$
\begin{array}{cl}
\text { Analysis equation: } & X_{k}=\frac{1}{T_{0}} \int_{-T_{0} / 2}^{T_{0} / 2} \tilde{x}(t) \exp \left(-j k \omega_{0} t\right) \mathrm{d} t, \\
\text { Synthesis equation: } & \tilde{x}(t)=\sum_{k=-\infty}^{\infty} X_{k} \exp \left(j k \omega_{0} t\right),
\end{array}
$$

According to the Dirichlet-Jordan Theorem [15], the above representation for a signal $\tilde{x}(t)$ is available provided it satisfies the following two DCs:

(A1) The signal $\tilde{x}(t)$ is absolutely integrable over one time period, i.e.,

$$
\int_{-T_{0} / 2}^{T_{0} / 2}|\tilde{x}(t)| \mathrm{d} t<\infty .
$$

(A2) It has BV over one time period. Signals with BV have (i) at most countably infinite number of maxima and minima, and (ii) at most countably infinite number of finite discontinuities. This is to note that vice-versa need not be true because signals with BV form a subset of signals satisfying (i) and/or (ii).

However, the signal processing and other literature [1-10] states the following three DCs over one time period:

(B1) The signal $\tilde{x}(t)$ is absolutely integrable, i.e.,

$$
\int_{-T_{0} / 2}^{T_{0} / 2}|\tilde{x}(t)| \mathrm{d} t<\infty .
$$

(B2) It has a finite number of maxima and minima. 
(B3) It has a finite number of discontinuities of finite magnitude.

Furthermore, the first DC is stated as the weak Dirichlet condition, while the second and third DCs are stated as strong Dirichlet conditions [4].

The first DC is stated identically in the literature, i.e., (A1) and (B1) are identical, and ensures the convergence of the integral leading to the computation of FS coefficients (8). In other words, it ensures the existence of the FS coefficients or the analysis equation.

Signals that satisfy second DC as stated originally (A2) with bounded variations over one time period, also satisfy the conditions: (a) at most countably infinite maxima and minima, and/or (b) at most countably infinite number of finite discontinuities. In fact, (A2) is required to ensure the recovery of the original signal using the Fourier coefficients (except at the points of discontinuities) via the synthesis equation (9). In other words, it ensures the convergence of FS (9).

The signal processing textbooks lack a clear explanation in multiple ways as listed below:

1. Superficially, the two sets of conditions (A2) vis-à-vis (B2)-(B3) may look similar, but they are not identical. Signals that satisfy (B2)-(B3) have bounded variations. But there may be signals that have bounded variations with at most countable infinite maxima and minima, and countable infinite discontinuities. Thus, the restriction to finite maxima and minima, and finite number of discontinuities incorrectly eliminates some signals that will have convergent FS.

2. While the first condition implies that signals belong to the $L_{1}$ space, the second condition (A2) is needed for only these signals belonging to the $L_{1}$ space. Interestingly, according to the Hunt theorem $[13,14]$, DCs are not required for signals that belong to $L_{p}$ with $p>1$. This is the reason that these conditions are stated to be sufficient but not necessary conditions. Further, the space $L_{1}$, i.e., $p=1$ is excluded from the Hunt theorem because, in 1925, Kolmogorov provided an example of a periodic signal $\tilde{x} \in \mathrm{L}_{1}(0,2 \pi)$ that satisfies (A1), but its FS diverges everywhere $[13,14]$.

3. It may not be appropriate to say that the first condition (B1) is weak and the second and third DCs (B2)-(B3) are strong conditions. Both the original DCs (A1) and (A2) are required for the signals belonging to $L_{1}$ and they have their own significance in terms of the analysis and synthesis of signals using the Fourier basis.

Further, it is to be noted that one can do analysis using the Fourier tool for signals that satisfy (A1), but fail (A2). Researchers may find use cases of such signals in applications, where only analysis using Fourier tools is helpful and synthesis back to the original signal is not required. For example, two popular continuous wavelets, Morlet and Maxican-Hat are not invertible, but they find immense applications in the analysis of geophysical signals [16-18]. 
From the above discussion, it is evident that the concept of convergence of FS is required to be added with better clarity to the textbooks for academicians, students, and researchers. In the next section, we will show examples from popular signal processing literature that satisfy (B1) but violate (B2) or (B3) and hence, are stated to fail FS convergence. However, these signals satisfy (A2) and hence, their FS would converge. These examples can easily highlight the distinction between (A2) and (B2)-(B3).

\section{Convergence of the Fourier series}

In this section, the results related to the convergence of FS are presented. Generally, three types of convergence, namely, point-wise, uniform, and norm convergence are important and widely discussed in the literature. These definitions of convergence are connected with how the partial sum [15]

$$
S_{N}(t)=\sum_{k=-N}^{N} X_{k} \exp \left(j k \omega_{0} t\right)
$$

converges to $\tilde{x}(t)$ as $N \rightarrow \infty$, i.e., whether $\lim _{N \rightarrow \infty} S_{N}(t)=\tilde{x}(t)$ is valid in some sense of convergence or not. The pointwise, uniform, and $L_{p}$-norm convergence are defined as

$$
\begin{array}{r}
S_{N} \rightarrow \tilde{x} \text { pointwise, if } \lim _{N \rightarrow \infty} S_{N}(t)=\tilde{x}(t), \quad \forall t \in\left[0, T_{0}\right], \\
S_{N} \rightarrow \tilde{x} \text { uniformly, if } \lim _{N \rightarrow \infty}\left\{\sup _{t \in\left[0, T_{0}\right]}\left|\tilde{x}(t)-S_{N}(t)\right|\right\}=0, \\
\text { and } S_{N} \rightarrow \tilde{x} \text { in } L_{p} \text { norm, if } \lim _{N \rightarrow \infty} \frac{1}{T_{0}} \int_{0}^{T_{0}}\left|\tilde{x}(t)-S_{N}(t)\right|^{p} \mathrm{~d} t=0,
\end{array}
$$

where $p \in \mathbb{R}, p \geq 1$ and $L_{p}$ norm is defined as

$$
\|\tilde{x}\|_{p}=\left[\frac{1}{T_{0}} \int_{0}^{T_{0}}|\tilde{x}(t)|^{p} \mathrm{~d} t\right]^{1 / p} .
$$

Theorem 1 (Dirichlet-Jordan): If $\tilde{x}$ has period $T_{0}$ and has bounded variation on $\left[0, T_{0}\right]$, then the Fourier series for $\tilde{x}$ converges for all values of $t$ as

$$
\lim _{N \rightarrow \infty} S_{N}(t)=\frac{1}{2}[\tilde{x}(t+)+\tilde{x}(t-)]
$$

where this convergence is uniform on every closed interval on which $\tilde{x}$ is continuous.

Theorem 2 (Parseval's equality): if $\frac{1}{T_{0}} \int_{-T_{0} / 2}^{T_{0} / 2}|\tilde{x}(t)|^{2} \mathrm{~d} t$ is finite, then

$$
\sum_{k=-\infty}^{\infty}\left|X_{k}\right|^{2}=\frac{1}{T_{0}} \int_{-T_{0} / 2}^{T_{0} / 2}|\tilde{x}(t)|^{2} \mathrm{~d} t .
$$


The convergence of FS for an $L_{2}$-function along with Riesz and Fischer theorem [15] provides necessary and sufficient conditions for $L_{2}$-convergence.

Theorem 3: If $\tilde{x}$ is an $L_{2}$-function on $\left[0, T_{0}\right]$, then its Fourier series converges to $\tilde{x}$ in $L_{2}$-norm. Conversely, If $\sum\left|X_{k}\right|^{2}$ converges, then there exists an $L_{2}$ function $\tilde{x}$ which has Fourier coefficients $\left\{X_{k}\right\}$.

The next theorem was proved by Carleson in 1966 [11] for $L_{2}$-functions, and was extended by Hunt in 1968 [12] for $L_{p}$ functions for all $p \in(1, \infty)$.

Theorem 4 (Carleson [11]): For each $\tilde{x} \in L_{2}\left(\left[0, T_{0}\right]\right)$, the Fourier series $S_{N}(t)$ converges to $\tilde{x}$ almost everywhere.

Theorem 5 (Hunt [12]): If $\tilde{x}$ is an $L_{p}$-function for $p>1$ on $\left[0, T_{0}\right]$, then its Fourier series converges to it at almost all points.

The phrases about convergence at 'almost all points' or 'almost everywhere' imply that the FS converges everywhere except at a countable set of points. Since the Lebesgue measure is zero on the set of countable points, the signal $\tilde{x}(t)$ is said to be equal to its series representation (Synthesis equation (9)) almost everywhere (excluding those countable points at which the divergence occurs). Thus, the convergence at 'almost all points' or 'almost everywhere' implies pointwise convergence at all but a set of countable points where divergence occurs. This convergence is a weaker version of pointwise convergence. It should also be noted that the condition of BV is not specified in Theorems 2-5. In the following subsections, we present some examples that are widely used in the signal processing literature to illustrate signals that fail (B2) or (B3) and are incorrectly stated as not having convergent FS representation [1-4].

\section{Case 1: A function that satisfies only the first DC (A1/B1)}

\section{Example-4}

Consider a signal $x(t)=\sin \left(\frac{2 \pi}{t}\right)$ for $0<t \leq 1$ and zero otherwise, as shown in Fig. 1 (top left) and use it to construct a periodic signal with fundamental period $(0,1]$ as

$$
\tilde{x}(t)=\sum_{m=-\infty}^{\infty} x(t-m)
$$

Clearly, $x(t) \notin B V([0,1])$ because $V(x,[0,1])=\infty$. However, $x(t) \in L_{p}(\mathbb{R})$ for $p \in[1, \infty]$. It has finite energy because $\int_{0}^{1}\left[\sin ^{2}\left(\frac{2 \pi}{t}\right)+\cos ^{2}\left(\frac{2 \pi}{t}\right)\right] \mathrm{d} t=1$. Thus, it lies in $L_{2}(\mathbb{R})$. Therefore, by Theorems $2-5$, its FS converges to the original signal at almost all points, although this signal is incorrectly stated as not having convergent FS representation. We compute the Fourier coefficients as

$$
X_{k}=\int_{0}^{1} \sin \left(\frac{2 \pi}{t}\right) e^{-j k \omega_{0} t} \mathrm{~d} t
$$


where $\omega_{0}=2 \pi \mathrm{rad} / \mathrm{s}$. By substituting $t=e^{-v}$, we obtain

$$
X_{k}=\int_{0}^{\infty} e^{-v} \sin \left(2 \pi e^{v}\right) e^{-j k \omega_{0} e^{-v}} \mathrm{~d} v,
$$

which is integration of an exponentially decaying function. One may observe from (17) and (18) that its Fourier coefficients are upper bounded to one, i.e., $\left|X_{k}\right| \leq 1$. Although its closed form expression is difficult to obtain, we used the numerical integration to compute the Fourier coefficients as shown in Fig. 1 (bottom left). For the purpose of illustration, Fig. 1 (right) depicts the corresponding plots obtained for the signal $x(t)=\cos \left(\frac{2 \pi}{t}\right), 0<t \leq 1$.
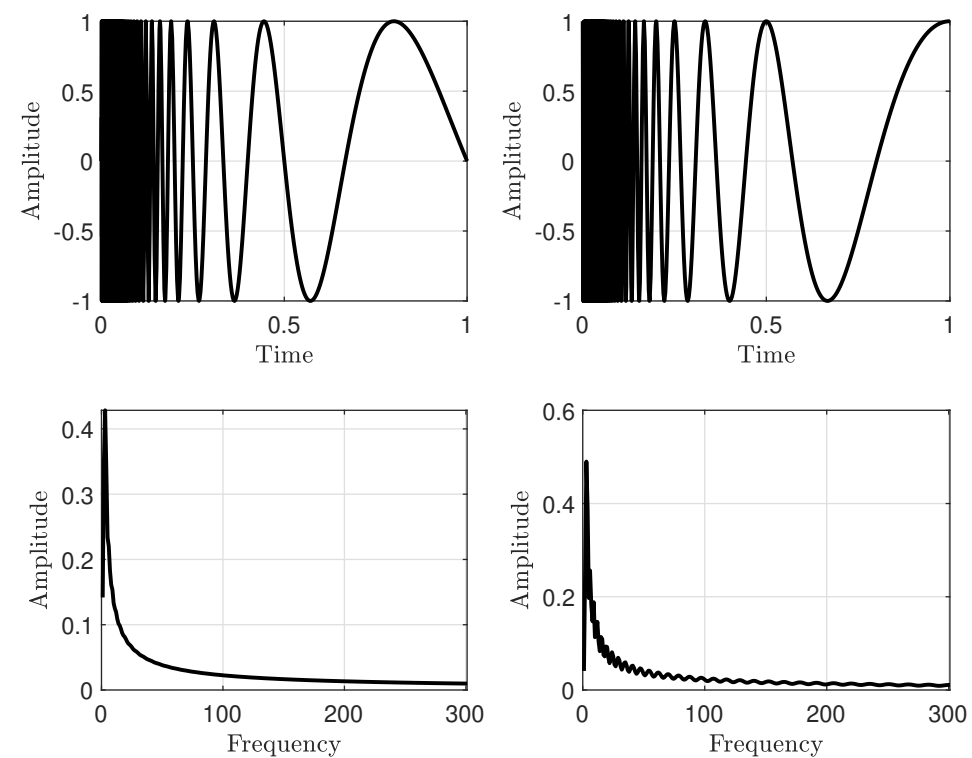

Figure 1: Plot of one period of signals over the time interval $0<t \leq 1$ and corresponding spectrum: (i) signal $\sin \left(\frac{2 \pi}{t}\right)$ (top left) and its spectrum (bottom left), (ii) signal $\cos \left(\frac{2 \pi}{t}\right)$ (top right) and its spectrum (bottom right).

\section{Example-5}

We consider another interesting example of a periodic signal with fundamental period $(0,1]$ as

$$
\tilde{x}(t)=\sin (\tan (\pi t)),
$$

as shown in bottom Fig. 2. Clearly, in one period, $\tilde{x}(t) \in L_{p}(\mathbb{R})$ for $p \geq 1$, as $\int_{0}^{1}|\sin (\tan (\pi t))|^{p} \mathrm{~d} t \leq \int_{0}^{1}|1|^{p} \mathrm{~d} t=1, \int_{0}^{1}\left[\sin ^{2}(\tan (\pi t))+\cos ^{2}(\tan (\pi t))\right] \mathrm{d} t=1$, which proves $\tilde{x}(t) \in L_{2}(\mathbb{R})$, but $\tilde{x}(t) \notin B V([0,1])$ as $\tilde{x}^{\prime}(t)=\pi \cos (\tan (\pi t)) \sec ^{2}(\pi t)$ and $V(x,[0,1])=\int_{0}^{1}\left|\tilde{x}^{\prime}(t)\right| \mathrm{d} t=\infty$. Theorems $2-5$ lead to convergence in this 

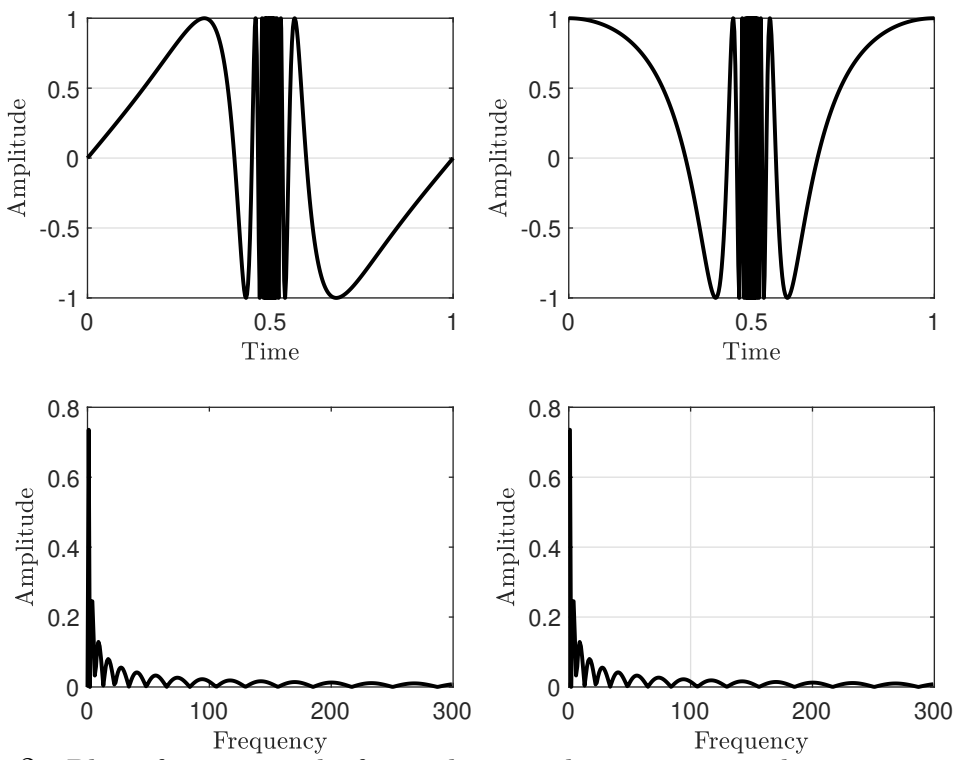

Figure 2: Plot of one period of signals over the time interval $0<t \leq 1$ and corresponding spectrum: (i) signal $\sin (\tan (\pi t))$ (top left) and its spectrum (bottom left), (ii) signal $\cos (\tan (\pi t))$ (top right) and its spectrum (bottom right).

case. Its Fourier coefficients can be obtained as

$$
\begin{aligned}
X_{k} & =\int_{0}^{1} \sin (\tan (\pi t)) e^{-j k \omega_{0} t} \mathrm{~d} t \\
\left|X_{k}\right| & =\left|\int_{0}^{1} \sin (\tan (\pi t)) e^{-j k \omega_{0} t} \mathrm{~d} t\right| \\
\left|X_{k}\right| & \leq \int_{0}^{1}\left|\sin (\tan (\pi t)) e^{-j k \omega_{0} t}\right| \mathrm{d} t \\
& \leq \int_{0}^{1} 1 \mathrm{~d} t=1
\end{aligned}
$$

thus they are bounded, i.e. $\left|X_{k}\right| \leq 1$, for all $k$. Although its closed form expression is difficult to obtain, we used the numerical integration to compute the Fourier coefficients as shown in Fig. 2 (bottom left). Similar analysis has been performed for the signal $\tilde{x}(t)=\cos (\tan (\pi t))$ and plots are shown in Fig. 2 (top right and bottom right). 


\section{Example-6}

Here, we consider a signal $x(t)=\frac{1}{\sqrt{t}}$ for $0<t \leq 1$, and zero otherwise. Now, we construct a periodic function

$$
\tilde{x}(t)=\sum_{m=-\infty}^{\infty} x(t-m),
$$

as shown in bottom Fig. 3. Clearly, $x(t) \in L_{1}(\mathbb{R})$ as $\int_{0}^{1} \frac{1}{\sqrt{t}} \mathrm{~d} t=2$, but $x(t) \notin$ $L_{2}(\mathbb{R})$, and $x(t) \notin B V([0,1])$ as $V(x,[0,1])=x(0)-x(1)=\infty$. However, $x(t) \in L_{p}(\mathbb{R})$ for $1 \leq p<2$. From Theorems $1-4$, it seems that the FS does not converge while Theorem 5 leads to the convergence in this case. Its Fourier coefficients can be obtained as

$$
\begin{aligned}
& X_{k}=\int_{0}^{1} \frac{1}{\sqrt{t}} e^{-j k \omega_{0} t} \mathrm{~d} t, \\
& X_{k}=\int_{0}^{1} 2 e^{-j k \omega_{0} z^{2}} \mathrm{~d} z,
\end{aligned}
$$

and they are bounded as $\left|X_{k}\right| \leq 2, \forall k$ and $X_{0}=2$. Although its closed form expression is difficult to obtain, we used the numerical integration to compute the Fourier coefficients as shown in Fig. 3 (bottom right).
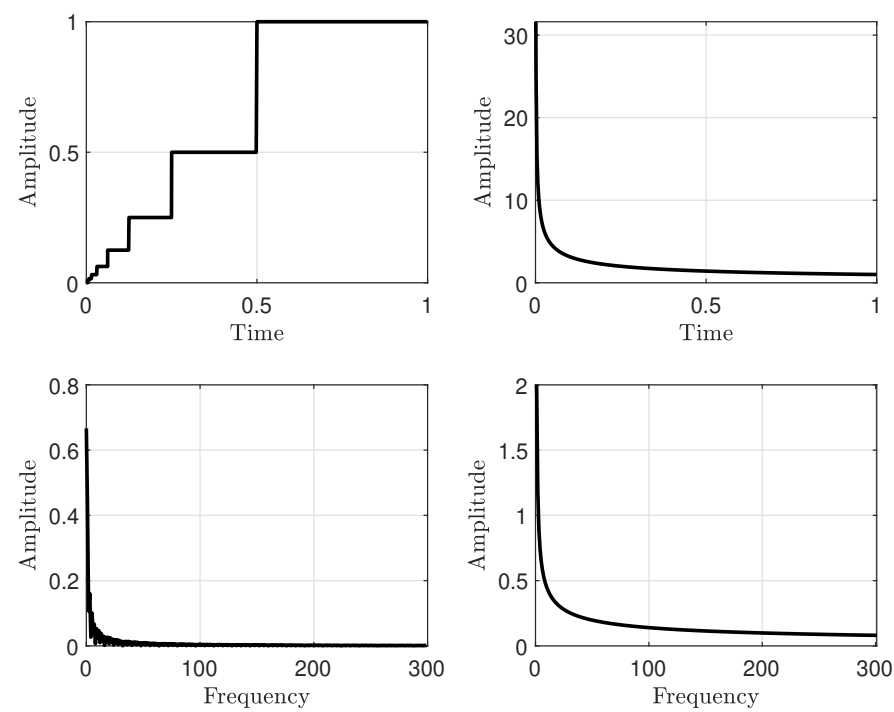

Figure 3: Plot of one period of signals and corresponding spectrum: (i) staircase signal (25) (top left) and its spectrum (bottom left), (ii) signal $\frac{1}{\sqrt{t}}$ (top right) and its spectrum (bottom right) 


\section{Case 2: A function that satisfies both the DCs (A1 and A2)}

\section{Example-7}

Here, we consider a series $x_{n}(t)=\frac{1}{2^{n}}$ for $\frac{1}{2^{n+1}} \leq t<\frac{1}{2^{n}}, n=0,1,2, \ldots$, and zero otherwise. Now, we construct a periodic signal

$$
\tilde{x}(t)=\sum_{m=-\infty}^{\infty} x(t-m), \text { where } x(t)=\sum_{n=0}^{\infty} x_{n}(t),
$$

as shown in top Fig. 3. This signal is also incorrectly stated as not having convergent FS representation since it does not satisfy DCs (B2 and B3). Clearly, $x(t) \in L_{p}(\mathbb{R})$ for $p \in[1, \infty]$, and $x(t) \in B V([0,1])$ as $V(x,[0,1])=1$. Using Theorems $1-5$, its FS converges to it at almost all points.

We compute the Fourier coefficients as

$$
\begin{aligned}
X_{k}= & \sum_{n=0}^{\infty} \int_{\frac{1}{2^{n+1}}}^{\frac{1}{2^{n}}} \frac{1}{2^{n}} e^{-j k \omega_{0} t} \mathrm{~d} t, \\
= & \frac{j}{k \omega_{0}} \sum_{n=0}^{\infty} \frac{1}{2^{n}}\left(e^{\frac{-j k \omega_{0}}{2^{n}}}-e^{\frac{-j k \omega_{0}}{2^{n+1}}}\right), \quad k \neq 0, \\
& \text { and } \quad X_{0}=\sum_{n=0}^{\infty} \int_{\frac{1}{2^{n+1}}}^{\frac{1}{2^{n}}} \frac{1}{2^{n}} \mathrm{~d} t=\frac{2}{3},
\end{aligned}
$$

where $\omega_{0}=2 \pi \mathrm{rad} / \mathrm{s}$. While its difficult to obtain a closed form expression, we observe from (26) that Fourier coefficients are upper bounded as

$$
\begin{aligned}
\left|X_{k}\right| & =\left|\frac{j}{k \omega_{0}} \sum_{n=0}^{\infty} \frac{1}{2^{n}}\left(e^{\frac{-j k \omega_{0}}{2^{n}}}-e^{\frac{-j k \omega_{0}}{2^{n+1}}}\right)\right|, \quad k \neq 0, \\
& \leq \frac{1}{|k| \omega_{0}} \sum_{n=0}^{\infty} \frac{1}{2^{n}}\left|\left(e^{\frac{-j k \omega_{0}}{2^{n}}}-e^{\frac{-j k \omega_{0}}{2^{n+1}}}\right)\right|, \quad k \neq 0, \\
& \leq \frac{1}{|k| \omega_{0}} \sum_{n=0}^{\infty} \frac{1}{2^{n}}\left(\left|e^{\frac{-j k \omega_{0}}{2^{n}}}\right|+\left|e^{\frac{-j k \omega_{0}}{2^{n+1}}}\right|\right)=\frac{4}{|k| \omega_{0}} .
\end{aligned}
$$

Hence, $\left|X_{k}\right| \leq \frac{4}{|k| \omega_{0}}=\frac{2}{|k| \pi}$ for $k= \pm 1, \pm 2, \ldots$, and $X_{0}=\frac{2}{3}$.

One may also argue that if FS of (16) and (25) do not converge, then they will create exceptions (counter-examples) to the well established results in Theorem 3, Theorem 4 and Theorem 5, and thus invalidate them.

For the sake of completeness, we provide some examples that neither satisfy (both) DCs, nor the theorem by Hunt. Therefore, their FS do not converge. Say, for example,

(i) $x_{1}(t)=\frac{1}{t}, \quad t \in(0,1]$ 
(ii) $x_{2}(t)=\frac{1}{t} \sin \left(\frac{2 \pi}{t}\right), \quad t \in(0,1]$

(iii) $x_{3}(t)=\sum_{n=0}^{\infty} x_{n}(t)$, where $x_{n}(t)=a^{n}$ for $\frac{1}{c^{n+1}} \leq t<\frac{1}{c^{n}}, c>1$, $n=0,1,2, \ldots, \infty$, diverges for $|a| \geq c$ and converges for $0 \leq|a|<c$.

\section{Dirichlet Conditions for Fourier Transform}

Fourier transform (FT) is the limiting case of Fourier series as the time period $T_{0}$ of a periodic signal $\tilde{x}(t)$ approaches $\infty$. In that case, the signal becomes aperiodic and the Fourier transform is the limiting case of the envelope of Fourier series. The Fourier transform (FT) and inverse FT are defined as [1-10]

$$
\begin{array}{ll}
\text { Analysis Equation: } \quad X(f)=\int_{-\infty}^{\infty} x(t) \mathrm{e}^{-j 2 \pi f t} \mathrm{~d} t, \\
\text { Synthesis Equation: } \quad x(t)=\int_{-\infty}^{\infty} X(f) \mathrm{e}^{j 2 \pi f t} \mathrm{~d} f,
\end{array}
$$

respectively. Similar to FS, the DCs for FT can be stated as below:

(C1) The signal $x(t)$ is absolutely integrable, i.e.,

$$
\int_{-\infty}^{\infty}|x(t)| \mathrm{d} t<\infty
$$

(C2) It has BV over $t \in(-\infty, \infty)$, i.e., the signal $x(t) \in B V(\mathbb{R})$. Such signals will have (i) at most countably infinite number of maxima and minima, and (ii) at most countably infinite number of finite discontinuities.

However, the DCs for FT are defined in the signal processing, communication and other literature as [1-10]:

(D1) The signal $x(t)$ is absolutely integrable, i.e.,

$$
\int_{-\infty}^{\infty}|x(t)| \mathrm{d} t<\infty
$$

(D2) It has a finite number of maxima and minima within any finite interval.

(D3) It has a finite number of finite discontinuities within any finite interval.

These DCs are stated to be the sufficient, but not necessary conditions for the convergence of FT. Similar to the subtle gaps observed in the statements of DCs of FS, (A2) vis-à-vis (B2)-(B3), we observe a few gaps in (C2) vis-àvis (D2)-(D3). Firstly, signals that satisfy (D2)-(D3) have bounded variations. But there may be signals that have bounded variations with at most countable infinite maxima and minima, and countable infinite discontinuities. Thus, the restriction to finite maxima and minima, and finite number of discontinuities incorrectly eliminates some signals that will have convergent FT. For example, 
FT of the signals $x(t)$ defined in (4) and (25) exist because they satisfy (C1) and $(\mathrm{C} 2)$. However, they are incorrectly stated as not having convergent FT because they do not satisfy (D2)-(D3). Secondly, there exist signals with valid FT representation, despite not satisfying DCs (C1) and/or (C2). For example, if $x(t) \in L_{2}(\mathbb{R})$, then its FT converges in $L_{2}$-norm [1-4]. Next, we present some interesting examples in support of the above statements regarding DCs.

\section{Example-8}

We consider a signal $x(t)=\sin \left(\frac{2 \pi}{t}\right)$ for $-\infty<t<\infty$, which does not satisfy DCs, i.e., $x(t) \notin L_{1}(\mathbb{R})$ and $x(t) \notin B V(\mathbb{R})$. This signal is incorrectly stated as not having convergent FT representation. Because $x(t) \in L_{2}(\mathbb{R})$, its FT converges in $L_{2}$-norm. One may also note that $x(t) \in L_{\infty}(\mathbb{R})$. To show that $x(t) \in L_{2}(\mathbb{R})$, we compute its energy $E_{x}$ as

$$
E_{x}=\int_{-\infty}^{\infty} \sin ^{2}\left(\frac{2 \pi}{t}\right) \mathrm{d} t=2 \int_{0}^{\infty} \sin ^{2}\left(\frac{2 \pi}{t}\right) \mathrm{d} t .
$$

On substituting $2 \pi / t=v$, we obtain

$$
E_{x}=4 \pi \int_{0}^{\infty}\left(\frac{\sin (v)}{v}\right)^{2} \mathrm{~d} v=2 \pi^{2} .
$$

The Fourier transform of this signal is given by

$$
X(f)=\int_{-\infty}^{\infty} \sin \left(\frac{2 \pi}{t}\right) \mathrm{e}^{-j 2 \pi f t} \mathrm{~d} t
$$

and numerical integration can be used to compute the spectrum.

\section{Example-9}

Interestingly, in contrast to the signal $x(t)=\sin \left(\frac{2 \pi}{t}\right)$ for $-\infty<t<\infty$ of Example-8, the signal $x(t)=\cos \left(\frac{2 \pi}{t}\right)$ for $-\infty<t<\infty$ does not satisfy DCs. Also, its FT does not converge because $x(t)$ does not belong to $L_{p}(\mathbb{R}), p \in[1, \infty)$, but belongs to only $L_{\infty}(\mathbb{R})$. We compute its energy $E_{x}$ as

$$
E_{x}=\int_{-\infty}^{\infty} \cos ^{2}\left(\frac{2 \pi}{t}\right) \mathrm{d} t=2 \int_{0}^{\infty} \cos ^{2}\left(\frac{2 \pi}{t}\right) \mathrm{d} t .
$$

The above energy is not finite because

$$
\begin{gathered}
\int_{-\infty}^{\infty}\left[\sin ^{2}\left(\frac{2 \pi}{t}\right)+\cos ^{2}\left(\frac{2 \pi}{t}\right)\right] \mathrm{d} t=\int_{-\infty}^{\infty} 1 \mathrm{~d} t=\infty \\
\text { and thus, } \quad \int_{-\infty}^{\infty} \cos ^{2}\left(\frac{2 \pi}{t}\right) \mathrm{d} t=\infty
\end{gathered}
$$


One can also observe that

$$
\int_{a}^{b} \cos ^{2}\left(\frac{2 \pi}{t}\right) \mathrm{d} t+\int_{a}^{b} \sin ^{2}\left(\frac{2 \pi}{t}\right) \mathrm{d} t=(b-a),
$$

which is finite for any finite duration $a \leq t \leq b$. Intuition regarding the above computations of energies can be easily made from the graph of the signals $\sin \left(\frac{2 \pi}{t}\right)$ and $\cos \left(\frac{2 \pi}{t}\right)$ plotted on the time interval $1 \leq t \leq 100$ as shown in the Fig. 4 top and bottom, respectively.
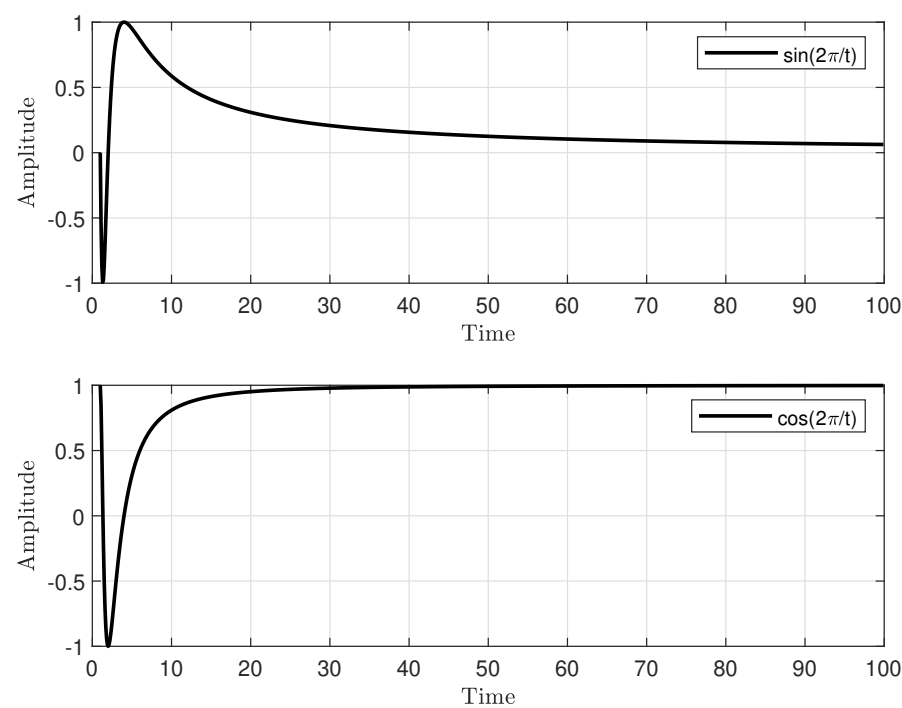

Figure 4: Plot of the signals $\sin \left(\frac{2 \pi}{t}\right)$ and $\cos \left(\frac{2 \pi}{t}\right)$ on the time interval $1 \leq t \leq 100$.

\section{Example-10}

Similar to Example-8, there is another interesting non-periodic signal that is frequently used, i.e., $x(t)=\frac{\sin (\pi t)}{\pi t}$ for $-\infty<t<\infty$, where $x(t) \notin L_{1}(\mathbb{R})$ and $x(t) \notin B V(\mathbb{R})$, i.e., it does not satisfy DCs. However, it is an $L_{2}(\mathbb{R})$ function. Its energy $\left(E_{x}\right)$ and FT can be obtained as

$$
\begin{aligned}
& E_{x}=\int_{-\infty}^{\infty}\left(\frac{\sin (\pi t)}{\pi t}\right)^{2} \mathrm{~d} t=1, \\
& X(f)= \begin{cases}1, & \text { if }-1 \leq f \leq 1, \\
0, & \text { otherwise }\end{cases}
\end{aligned}
$$

The two alternative sets of requirements, i.e., (i) fulfillment of DCs, (ii) finite energy, are sufficient to guarantee that a signal has a Fourier representation. The set of signals which are neither absolutely integrable nor square integrable over an infinite interval, can have Fourier representation if Dirac delta impulse function [19] is included in the Fourier representation [1-4]. 


\section{Some Concluding Remarks}

In this lecture note, we have developed a clear understanding of the Dirichlet conditions with detailed discussions and suitable examples. The clarifications provided here are not available across many popular books, articles, and lecture notes. Also, some of the examples considered in this note are inadvertently used in the existing literature to illustrate signals not having a valid Fourier representation. The Fourier analysis for these widely-used examples has been presented here to reveal some new findings. Therefore, as an outcome of this study, appropriate clarifications may be added to the existing literature to ensure proper dissemination of the intended information to students and researchers working across a plethora of applications utilizing Fourier theory.

\section{References}

[1] A.V. Oppenheim, A.S. Willsky, S.H. Nawab, Signals and Systems-2nd ed. 1996, Prentice-Hall, Inc. Simon \& Schuster/A Viacom Company Upper Saddle River, New Jersey 07458, ISBN 0-13-814757-4.

[2] J.G. Proakis, D.G. Manolakis, Digital Signal Processing Principles, Algorithms, and Applications-3rd ed. 1996, Prentice-Hall, Inc. Simon \& Schuster/A Viacom Company Upper Saddle River, New Jersey 07458, ISBN 0-13-394338-9.

[3] S. Haykin, M. Moher, Introduction to Analog and Digital Communications, 2007 John Wiley \& Sons, Inc. ISBN-13 978-0-471-43222-7.

[4] B.P. Lathi, Signal Processing and Linear Systems, 2000, BerkeleyCambridge Press, US ISBN-13: 978-0941413350.

[5] P. Singh, S. D. Joshi, R. K. Patney, K. Saha, "The Fourier decomposition method for nonlinear and non-stationary time series analysis," Proc. R. Soc. A 20160871, 2017.

[6] J.C.R. Hunt, J. C. Vassilicos, "Kolmogorov's contributions to the physical and geometrical understanding of small-scale turbulence and recent developments," Proc. R. Soc. Lond. A 434183-210, 1991. http://doi.org/10. 1098/rspa.1991.0088

[7] H. Zhao, D. Zhang, H. Meng, J. Tao, "Analytical measurement method for situation elements' dynamic characteristics," Journal of Systems Engineering and Electronics Vol. 28, No. 6, pp. 1126-1132, 2017.

[8] G.A. Gibson, "On the History of the Fourier Series," pp. 137-166, Seventh Meeting, May 12, 1893. https://www. cambridge.org/core/services/aop-cambridge-core/content/ view/2E133C9A3EB69055C7CD01F0E0D79BD3/S001309150003131Xa.pdf/ div-class-title-on-the-history-of-the-fourier-series-div.pdf 
[9] Qiming Chen, Xun Lang, Lei Xie, Hongye Su, Multivariate intrinsic chirp mode decomposition, Signal Processing, vol. 183, 2021, 108009, https: //doi.org/10.1016/j.sigpro.2021.108009.

[10] K. Eren, "Spectral analysis of GEOS-3 altimeter data and frequency domain collocation," Ph.D. dissertation, The Ohio State University, 1980.

[11] L. Carleson, "On convergence and growth of partial sums of Fourier series," Acta Math 116, pp 137-157, 1966.

[12] R.A. Hunt, "On the convergence of Fourier series, Orthogonal Expansions and their Continuous Analogues," Proc. Conf., Edwardsville, Ill., 1967, Southern Illinois Univ. Press, Carbondale, Ill., (1968), 235-255.

[13] A.N. Kolmogorov, "Une série de Fourier-Lebesgue divergente presque partout," Fundamenta Mathematicae, vol. 4, pp. 324-328, 1923, doi:10. 4064/fm-4-1-324-328.

[14] A.N. Kolmogorov, "Une série de Fourier-Lebesgue divergente partout," Comptes Rendus, 183, 1327-1328, 1926.

[15] J.S. Walker, Fourier series, Encyclopedia of Physical Science and Technology, Third Edition, Academic Press, 6 (2002), 167-183.

[16] M.S.Tiscareno and M.M. Hedman, "A review of Morlet wavelet analysis of radial profiles of Saturn's rings," Philosophical Transactions of the Royal Society A: Mathematical, Physical and Engineering Sciences, vol. 376, no. 2126, pp.1-36, 2018.

[17] P. Kumar and F.G. Efi, "Wavelet analysis for geophysical applications," Reviews of geophysics, vol. 35, no. 4, pp. 385-412, 1997.

[18] A.P. Shanker and R.S. Nanjundiah, "Morlet wavelet analysis of tropical convection over space and time: Study of poleward propagations of Intertropical Convergence Zone (ITCZ)," Geophysical Research Letters, vol. 31, no. 2, pp. 1-4, 2004.

[19] C. Candan, "Proper Definition and Handling of Dirac Delta Functions [Lecture Notes]," in IEEE Signal Processing Magazine, vol. 38, no. 3, pp. 186203, May 2021, doi: 10.1109/MSP.2021.3055025. 